\title{
Empirical Likelihood Confidence Band for the Difference of Survival Functions under Proportional Hazards Model
}

\author{
Mai Zhou, Shihong Zhu* \\ Department of Statistics, University of Kentucky, Lexington, Kentucky 40508, U.S.A.
}

\begin{abstract}
Under the stratified Cox model, an empirical likelihood based simultaneous confidence band for the difference of two individualized survival functions is proposed. Simulation studies demonstrate its superiority over the normal approximation based competitors.
\end{abstract}

Keywords: Chi-square distribution; Stratified Cox model; Two-sample comparison

\section{Introduction}

In biomedical studies, it is of great importance to compare two treatments that give rise to censored time-to-event data. The conventional approach relies on the popular Cox Proportional Hazards Model to estimate a constant hazard ratio as the treatment effect measure. However, the proportional hazards assumption is often violated in practice and accordingly many alternative treatment effect measures have been proposed. In particular, Zhang and Klein (2001) extended the work of Parzen et al. (1997) and obtained a Wald-type confidence band for the difference of two individualized survival functions with covariates adjusted by a stratified Cox model. As pointed out by Bie et al. (1987), such normal approximation based confidence band for hazard or survival functions related parameters may have unsatisfactory small sample performance and may include values outside the natural range of the parameters. Appropriate transformations are necessary to improve the coverage accuracy, but it is unclear what might serve as a successful transformation (Parzen et al., 1997). Motivated by the many desirable properties of the empirical likelihood (EL) method, in particular, its transformation respecting property that renders transformations unnecessary, under the same framework of Zhang and Klein (2001), we provide in this manuscript an EL based confidence band for the difference of two individualized survival functions.

As an appealing nonparametric method, EL has been widely used in the literature to compare two censored samples. EL inference on the difference of mean and median survival times has been studied in Wang and Wang (2001); EL simultaneous confidence bands have been obtained for Q-Q plots (Einmahl and McKeague, 1999), ratio of survival

\footnotetext{
* Corresponding Author

Email address: shihong. zhu@uky . edu (Shihong Zhu )
} 
functions (McKeague and Zhao, 2002), difference of survival functions (Shen and He, 2006), and ratio of instant haz-

For ease of presentation, we will focus on the case of $\boldsymbol{Z}_{0}=\mathbf{0}$ such that $\theta_{0}\left(t \mid \boldsymbol{Z}_{0}\right)$ is merely the difference of the baseline survival functions. When $\boldsymbol{Z}_{0} \neq \mathbf{0}$, we may use the shifted vector $\boldsymbol{Z}_{i j}-\boldsymbol{Z}_{0}$ as if it were originally observed. Due to this shift, the difference of the new baseline survival functions will be equal to the difference of the original survival functions with covariate $Z_{0}$. Therefore in what follows $Z_{0}$ is assumed to be $\mathbf{0}$ and we simply 
50 hypothesized value of $\theta_{0}(t)$ and $\eta=\eta(t)$ a hypothesized value of $S_{1}(t)$. We define the following EL ratio at $(\theta, \eta)$ :

$$
\Re(\theta, \eta, t)=\frac{\sup _{P_{1}, P_{2}}\left\{L\left(P_{1}, P_{2}, \widehat{\boldsymbol{\beta}}\right) \mid P_{1}(t)=-\log \eta, P_{2}(t)=-\log (\eta-\theta)\right\}}{\sup _{\boldsymbol{\beta}, P_{1}, P_{2}} L\left(P_{1}, P_{2}, \boldsymbol{\beta}\right)} .
$$

The restriction that $\eta \in \Theta \equiv(\max (0, \theta), \min (1,1+\theta))$ is imposed such that both $\eta$ and $\eta-\theta$ fall in $(0,1)$, the natural range of a survival probability. Two comments on the numerator in (2.3) are in order. Firstly, the introduction of the intermediate variable $\eta$ is essential to linearize the constraint. Earlier use of this linearization technique can be found in 70 Naik-Nimbalkar and Rajarshi (1997) and Shen and He (2006), among others. Secondly, when $\boldsymbol{\beta}$ is multidimensional, maximization over $\boldsymbol{\beta}$ in the presence of constraint could be numerically prohibitive. Therefore, we fix $\boldsymbol{\beta}$ at its partial likelihood estimator $\widehat{\boldsymbol{\beta}}$, eliminating the need for maximization over $\boldsymbol{\beta}$. Similar plug-in strategy of using an estimated 
parameter has been widely used in the literature (Dong and Matthews, 2012; Hjort et al., 2009).

${ }_{75}$ Let $g_{i j}=I\left[T_{i j} \leq t\right]$ and $S_{i j}=S_{i}^{(0)}\left(\widehat{\boldsymbol{\beta}}, T_{i j}\right)$. Simple calculation using Lagrange Multiplier method yields

$$
\log \Re(\theta, \eta, t)=-\sum_{i=1}^{2} \sum_{j=1}^{n_{i}} \delta_{i j} \log \left\{1+\frac{g_{i j}}{S_{i j}} \lambda_{i}\right\}-n\left\{\lambda_{1} \log \eta+\lambda_{2} \log (\eta-\theta)\right\},
$$

where the Lagrange multipliers $\lambda_{1}$ and $\lambda_{2}$ are implicit functions of $\eta, \theta$, and $t$, described by the following equations:

$$
\left\{\begin{array}{l}
\frac{1}{n} \sum_{j=1}^{n_{1}} \frac{\delta_{1 j} g_{1 j}}{S_{1 j}+\lambda_{1}}+\log \eta=0, \\
\frac{1}{n} \sum_{j=1}^{n_{2}} \frac{\delta_{2 j} g_{2 j}}{S_{2 j}+\lambda_{2}}+\log (\eta-\theta)=0 .
\end{array}\right.
$$

For any $\eta \in \Theta$, as long as $\sum_{j} \delta_{1 j} g_{1 j}>0$, using a monotonicity and continuity argument, we can show that there is a unique and smooth solution $\lambda_{1}$ to (2.5) such that $S_{1 j}+\lambda_{1}>0$ for all $j \in\left\{1,2, \cdots, n_{1}\right\}$. Similar claim regarding equation (2.6) can be also made. Moreover, taking derivative with respect to $\eta$ on both sides of (2.5) and (2.6) leads to

$$
\sum_{j=1}^{n_{1}} \frac{\delta_{1 j} g_{1 j}}{\left(S_{1 j}+\lambda_{1}\right)^{2}} \frac{\partial \lambda_{1}}{\partial \eta}=\frac{n}{\eta} \quad \text { and } \quad \sum_{j=1}^{n_{2}} \frac{\delta_{1 j} g_{2 j}}{\left(S_{2 j}+\lambda_{2}\right)^{2}} \frac{\partial \lambda_{2}}{\partial \eta}=\frac{n}{\eta-\theta} .
$$

In order to profile out the intermediate variable $\eta$, we equate with zero the derivative of (2.4) with respect to $\eta$. While maximizes $\Re\left\{\theta_{0}(t), \eta, t\right\}$ and converges uniformly in probability to $S_{1}(t)$, and $-2 \sigma^{2}(t) \log \Re\left\{\theta_{0}(t), \eta_{n}(t), t\right\} \stackrel{\mathscr{D}}{\longrightarrow}$ $U^{2}(t)$ in $D\left[\tau_{0}, \tau\right]$, where $U(t)$ is a mean-zero Gaussian process with covariance function $v(t, s)$ defined in $(2.1)$ and

$$
\sigma^{2}(t)=\sum_{i=1}^{2} S_{i}^{2}(t) \int_{0}^{t}\left\{s_{i}^{(0)}\left(\boldsymbol{\beta}_{0}, u\right)\right\}^{-1} \mathrm{~d} \Lambda_{i}(u) .
$$


Here $v(t, t)$ is the asymptotic variance of $\widehat{\theta}_{0}(t)$ while $\sigma^{2}(t)$ can be viewed as the variance of $\widehat{\theta}_{0}(t)$ when $\boldsymbol{\beta}_{0}$ is known. According to Zhang and Klein (2001), they can be consistently estimated by $\widehat{v}(t, t)$ and $\widehat{\sigma}^{2}(t)$ obtained by replacing the unknown $\boldsymbol{s}^{(k)}\left(\boldsymbol{\beta}_{0}, t\right)$ and $\Lambda_{i}$ in (2.1) and (2.9) with their empirical counterparts $\boldsymbol{S}^{(k)}(\widehat{\boldsymbol{\beta}}, t)$ and $\widehat{\Lambda}_{i}$ respectively.

Remark. Theorem 1 only asserts the existence of a solution to equation (2.8). It does not establish the uniqueness of the solution, which is why we can only characterize $\eta_{n}(t)$ as a local maximizer of $\Re\left\{\theta_{0}(t), \eta, t\right\}$, instead of a global one. However, the uniqueness was observed in all of our numeric studies (not shown here).

Remark. It follows from Theorem 1 that, at a fixed $t \in\left[\tau_{0}, \tau\right],-2 \log \Re\left\{\theta_{0}(t), \eta_{n}(t), t\right\} \stackrel{\mathscr{D}}{\rightarrow} v(t, t) / \sigma^{2}(t) \chi_{1}^{2}$, a scaled chi-square distribution with one degree of freedom. Let $\chi_{1}^{2}(\alpha)$ be the upper $\alpha$ percentile of $\chi_{1}^{2}$, then the level $\alpha$ EL confidence interval for $\theta_{0}(t)$ is given by $\left\{\theta \mid-2 \widehat{\sigma}^{2}(t) \log \Re\left\{\theta, \eta_{n}(t), t\right\} \leq \widehat{v}(t, t) \chi_{1}^{2}(\alpha)\right\}$.

\subsection{Confidence Band}

Theorem 1 provides us the basis of constructing the simultaneous EL confidence band for $\theta_{0}(t)$. Let $C_{\alpha}\left(\tau_{0}, \tau_{1}\right)$ be the upper $\alpha$ percentile of $\sup _{\tau_{0} \leq t \leq \tau} U^{2}(t) / v(t, t)$, then a level $\alpha$ EL simultaneous confidence band for $\theta_{0}(t)$ on $\left[\tau_{0}, \tau\right]$ is given by

$$
\left\{(\theta(t), t) \mid \sup _{\tau_{0} \leq t \leq \tau}-2 \widehat{\sigma}^{2}(t) \log \Re\left\{\theta(t), \eta_{n}(t), t\right\} \leq \widehat{v}(t, t) C_{\alpha}\left(\tau_{0}, \tau\right), t \in\left[\tau_{0}, \tau\right]\right\}
$$

Monte-Carlo technique is needed to estimate $C_{\alpha}\left(\tau_{0}, \tau\right)$. Since $U(t)$ does not have independent increment as clearly indicated by its covariance structure $v(t, s)$, it is not easy to generate its sample path. We could follow the MonteCarlo simulation strategy in Lin et al. (1994) to approximate the sample path of $U(t)$ based on a modification to the martingale representation of $\sqrt{n}\left\{\widehat{\theta}_{0}(t)-\theta_{0}(t)\right\}$. In this manuscript, we propose an alternative method that is similar to their approach but features a more transparent justification. The new method is based on the observation that $U(t)$ can be decomposed into several components each of which can be easily simulated. Specifically, let $\boldsymbol{G}$ be a multivariate normal random variable with covariance $\boldsymbol{\Sigma}^{-1}$ and $V_{i}(t)$ be a mean-zero Gaussian process independent of $\boldsymbol{G}$ with independent increment and variance function $\sigma_{i}^{2}(t)=\int_{0}^{t}\left\{s_{i}^{(0)}\left(\boldsymbol{\beta}_{0}, u\right)\right\}^{-1} \mathrm{~d} \Lambda_{i}(u)$, then the process $W(t)$ defined in the following has the same distribution with $U(t)$ :

$$
W(t)=\sum_{i=1}^{2} S_{i}(t) V_{i}(t)+\left\{S_{1}(t) \boldsymbol{h}_{1}(t)-S_{2}(t) \boldsymbol{h}_{2}(t)\right\}^{\top} \boldsymbol{G}
$$

Due to its independent increment property, $V_{i}(t)$ can be easily simulated by sequentially generating its normally distributed increments. Surely we also need to replace the unknown parameters $S_{i}(t), \boldsymbol{h}_{i}(t), \sigma_{i}^{2}(t)$ and $\boldsymbol{\Sigma}$ by their corresponding estimates $\widehat{S}_{i}(t), \widehat{\boldsymbol{h}}_{i}(t), \widehat{\sigma}_{i}^{2}(t)$, and $\widehat{\boldsymbol{\Sigma}}$ obtained by replacing in their definitions $\boldsymbol{s}^{(k)}\left(\boldsymbol{\beta}_{0}, t\right)$ and $\Lambda_{i}$ with $\boldsymbol{S}^{(k)}(\widehat{\boldsymbol{\beta}}, t)$ and $\widehat{\Lambda}_{i}$ respectively. We observe that $\widehat{\sigma}_{i}^{2}(t)$ is piecewise constant and only jumps at the observed failure times, therefore we only need to sample $V_{i}(t)$ at those observed failure times, which amounts to approximating $W(t)$ 
by the following process

$$
W^{*}(t)=\sum_{i=1}^{n} \sum_{j=1}^{n_{i}} \widehat{S}_{i}(t) \frac{\delta_{i j} I\left[T_{i j} \leq t\right] G_{i j}}{S_{i}^{(0)}\left(\widehat{\boldsymbol{\beta}}, T_{i j}\right)}+\left\{\widehat{\boldsymbol{h}}_{1}(t) \widehat{S}_{1}(t)-\widehat{\boldsymbol{h}}_{2}(t) \widehat{S}_{2}(t)\right\}^{\top} \boldsymbol{G}_{0},
$$

where $G_{i j}\left(i=1,2 ; j=1,2, \cdots, n_{i}\right)$ are i.i.d. standard normal random variables and $\boldsymbol{G}_{0}$ is a multivariate normal random variable with covariance $\widehat{\boldsymbol{\Sigma}}^{-1}$. We could generate, say $N$, sample paths of $W^{*}(t)$, for each of these sample paths calculate $c=\sup _{\tau_{0} \leq t \leq \tau} W^{* 2}(t) / \widehat{v}(t, t)$, and estimate $C_{\alpha}\left(\tau_{0}, \tau\right)$ by the upper $\alpha$ percentile of the resulting $c$ values. In practice, it is important to choose a sufficiently large $N$ to stabilize the estimate. In our simulation study, $N=5000$ appears to work very well. This is consistent with Lin et al. (1994) and Zhang and Klein (2001) who reported in their respective studies that $N$ of about a few thousand is enough for a $95 \%$ confidence band.

\section{Numerical Study}

\subsection{Simulation}

We assume the underlying survival time $X_{i j}$ have a Weibull distribution with cumulative hazard function

$$
\Lambda_{i j}\left(t \mid Z_{i j}\right)=\alpha_{i} t^{\gamma_{i}} \exp \left(Z_{i j}^{\top} \beta_{0}\right), \quad i=1,2 ; j=1,2, \cdots, n_{1} .
$$

In this setup, the proportional hazards assumption holds within each stratum but not across the strata if $\gamma_{1} \neq \gamma_{2}$. The parameter $\gamma_{i}$ controls the shape of the distribution of $X_{i j}$ and $\alpha_{i}$ adjusts its scale. The censoring times $C_{1 j}$ and $C_{2 j}$ are assumed to be exponentially distributed with mean $\theta_{1}$ and $\theta_{2}$, respectively. The univariate covariate $Z_{i j}$ is drawn from the uniform distribution on [0,1]. In our simulation study, $\gamma_{1}=1.1, \gamma_{2}=1, \alpha_{1}=0.5, \alpha_{2}=1$, and $\beta_{0}=0.1$. Finally, $\theta_{1}$ and $\theta_{2}$ are adjusted to incorporate various censoring rates in both groups. We apply both the EL and normal approximation confidence bands for $\theta_{0}(t)$ on the interval $\left[\tau_{0}, \tau\right]$, where $\tau_{0}$ is the first observed death in either stratum 1 or 2, whichever comes later; $\tau$ is chosen such that the risk set in each group contains at least 10 percent of the observations in the corresponding group. $C_{\alpha}\left(\tau_{0}, \tau\right)$ is estimated based on 5000 replicates.

Following the suggestion from one referee, we also considered the normal approximation method with two transformations that simply mimic the log-minus-log and arcsine-square-root transformations in Bie et al. (1987). Namely, we considered applying $f_{1}(x)=\log [-\log \{(x+1) / 2\}]$ and $f_{2}(x)=\arcsin \sqrt{(x+1) / 2}$ on $\widehat{\theta}_{0}(t)$. Observed coverage percentages of the confidence bands using 1000 simulation replicates are summarized in Table 1. Coverage percentages and average lengths of the confidence intervals for $\theta_{0}(2)$ are given in Table 2 and Table 3.

It can be seen from the tables that the EL method has a better overall performance than the normal approximation based alternatives. Specifically, the EL confidence band has accurate coverage when the censoring rate is less than or equal to $30 \%$. When the censoring rate reaches $50 \%$, it gains accuracy quickly as the sample size increases. While the normal approximation confidence band is clearly under-covering. The arcsine-square-root transformation seems 
to be able to slightly improve the coverage when the sample size is small while the log-minus-log transformation does not. In the case of confidence intervals, the advantage of the EL method is still perceivable, evidenced by the better coverage but similar average length achieved by the EL confidence intervals. Plots not shown here indicate that, when the sample size is small, the sampling distribution of $\widehat{\theta}_{0}(t)$ is clearly skewed, which might explain why the normal confidence interval fails to achieve the nominal coverage level, but it is less of a problem for the EL confidence interval, because one well-known advantage of the EL method is its ability to adapt to skewed sampling distribution and generate confidence intervals that have a data-driven shape.

Table 1: Observed coverage percentages of nominal $95 \%$ simultaneous confidence bands based on 1000 replicates. $C$ denotes the censoring rate and $n$ denotes the sample size per group.

\begin{tabular}{llllcccc}
\hline$\alpha$ & $\beta$ & $C$ & $n$ & Log-Log & Arcsine & Normal & Empirical \\
\hline 16 & 9.0 & $10 \%$ & 25 & 91.4 & 92.7 & 91.5 & 95.8 \\
& & & 30 & 91.3 & 92.9 & 91.6 & 95.1 \\
& & & 50 & 92.2 & 93.3 & 93.5 & 95.8 \\
& & & 100 & 93.7 & 94.2 & 94.0 & 95.4 \\
7.1 & 4.0 & $20 \%$ & 25 & 90.6 & 92.6 & 91.8 & 95.5 \\
& & & 30 & 91.9 & 92.7 & 92.2 & 95.1 \\
& & & 50 & 92.8 & 93.1 & 92.5 & 94.7 \\
4.3 & 2.2 & $30 \%$ & 25 & 90.6 & 92.4 & 91.1 & 95.1 \\
& & & 30 & 91.3 & 92.3 & 90.8 & 94.4 \\
& & & 50 & 92.5 & 92.9 & 92.3 & 94.8 \\
1.9 & 1.0 & $50 \%$ & 25 & 82.9 & 93.4 & 93.5 & 95.6 \\
& & & 30 & 89.3 & 90.8 & 89.2 & 93.4 \\
& & & 50 & 90.2 & 91.4 & 90.7 & 93.9 \\
& & & 100 & 92.1 & 92.7 & 92.1 & 94.1 \\
\hline
\end{tabular}

Table 3: Observed coverage percentages and $10^{4} \times$ average lengths (in parenthesis) of nominal $95 \%$ confidence intervals for $\theta_{0}(2)$ based on 5000 replicates. $C$ denotes the censoring rate $n_{i}$ denotes the sample size in the $i$ th group.

\begin{tabular}{lllllcccc}
\hline$\alpha$ & $\beta$ & $C$ & $n_{1}$ & $n_{2}$ & Log-Log & Arcsine & Normal & Empirical \\
\hline \multirow{2}{*}{16} & 9 & $10 \%$ & 25 & 50 & $93.86(4319)$ & $93.62(4304)$ & $93.28(4341)$ & $95.08(4293)$ \\
& & & 30 & 60 & $93.92(3957)$ & $93.70(3946)$ & $93.38(3974)$ & $94.72(3936)$ \\
& & & 50 & 100 & $94.64(3089)$ & $94.54(3084)$ & $94.52(3097)$ & $94.94(3078)$ \\
7.1 & 4 & $20 \%$ & 25 & 50 & $93.70(4573)$ & $93.72(4555)$ & $93.40(4600)$ & $95.26(4547)$ \\
& & & 30 & 60 & $93.68(4192)$ & $93.58(4177)$ & $93.28(4212)$ & $94.88(4170)$ \\
& & & 50 & 100 & $94.02(3278)$ & $94.04(3272)$ & $93.76(3287)$ & $94.76(3265)$ \\
4.3 & 2.2 & $30 \%$ & 25 & 50 & $93.92(4929)$ & $93.86(4907)$ & $93.34(4963)$ & $95.10(4904)$ \\
& & & 30 & 60 & $93.52(4520)$ & $93.56(4502)$ & $93.04(4545)$ & $94.78(4500)$ \\
& & & 50 & 100 & $94.64(3547)$ & $94.22(3539)$ & $94.02(3559)$ & $94.98(3534)$ \\
1.9 & 1.0 & $50 \%$ & 25 & 50 & $92.52(6026)$ & $92.64(5988)$ & $92.06(6100)$ & $94.52(5966)$ \\
& & & 30 & 60 & $93.00(5600)$ & $93.36(5569)$ & $92.90(5654)$ & $94.62(5542)$ \\
& & & 50 & 100 & $93.92(4482)$ & $93.98(4467)$ & $93.58(4509)$ & $94.82(4447)$ \\
\hline
\end{tabular}


Table 2: Observed coverage percentages and $10^{4} \times$ average lengths (in parenthesis) of nominal $95 \%$ confidence intervals for $\theta_{0}(2)$ based on 5000 replicates. $C$ denotes the censoring rate and $n_{i}$ denotes the sample size in the $i$ th group.

\begin{tabular}{|c|c|c|c|c|c|c|c|c|}
\hline$\alpha$ & $\beta$ & $C$ & $n_{1}$ & $n_{2}$ & $\log -\log$ & Arcsine & Normal & Empirical \\
\hline 16 & 9 & $10 \%$ & $\begin{array}{l}25 \\
30 \\
50 \\
100\end{array}$ & $\begin{array}{l}25 \\
30 \\
50 \\
100\end{array}$ & $\begin{array}{l}93.52(4833) \\
93.90(4442) \\
94.58(3463) \\
94.76(2449)\end{array}$ & $\begin{array}{l}93.56(4812) \\
94.04(4427) \\
94.52(3458) \\
94.90(2446)\end{array}$ & $\begin{array}{l}93.14(4865) \\
93.68(4468) \\
94.16(3475) \\
94.80(2453)\end{array}$ & $\begin{array}{l}95.00(4899) \\
94.84(4488) \\
94.90(3485) \\
95.10(2456)\end{array}$ \\
\hline 7.1 & 4 & $20 \%$ & $\begin{array}{l}25 \\
30 \\
50 \\
100\end{array}$ & $\begin{array}{l}25 \\
30 \\
50 \\
100\end{array}$ & $\begin{array}{l}93.28(5140) \\
94.04(4720) \\
94.34(3682) \\
94.78(2621)\end{array}$ & $\begin{array}{l}93.56(5116) \\
94.06(4710) \\
94.38(3674) \\
94.76(2618)\end{array}$ & $\begin{array}{l}93.28(5181) \\
93.70(4751) \\
94.22(3697) \\
94.66(2626)\end{array}$ & $\begin{array}{l}94.74(5208) \\
95.02(4772) \\
95.08(3707) \\
94.86(2629)\end{array}$ \\
\hline 4.3 & 2.2 & $30 \%$ & $\begin{array}{l}25 \\
30 \\
50 \\
100\end{array}$ & $\begin{array}{l}25 \\
30 \\
50 \\
100\end{array}$ & $\begin{array}{l}93.14(5568) \\
93.38(5111) \\
94.58(4046) \\
94.44(2869)\end{array}$ & $\begin{array}{l}93.36(5540) \\
93.56(5089) \\
94.36(4036) \\
94.60(2866)\end{array}$ & $\begin{array}{l}92.90(5624) \\
93.22(5153) \\
94.24(4066) \\
94.56(2876)\end{array}$ & $\begin{array}{l}94.68(5639) \\
94.94(5166) \\
95.04(4069) \\
95.02(2877)\end{array}$ \\
\hline 1.9 & 1.0 & $50 \%$ & $\begin{array}{l}25 \\
30 \\
50 \\
100\end{array}$ & $\begin{array}{l}25 \\
30 \\
50 \\
100\end{array}$ & $\begin{array}{l}89.48(6762) \\
90.34(6287) \\
91.96(5106) \\
94.34(3728)\end{array}$ & $\begin{array}{l}90.58(6727) \\
91.44(6256) \\
92.92(5089) \\
94.68(3715)\end{array}$ & $\begin{array}{l}90.18(6887) \\
91.10(6381) \\
92.52(5153) \\
94.44(3739)\end{array}$ & $\begin{array}{l}91.94(6778) \\
92.42(6296) \\
94.42(5093) \\
94.88(3709)\end{array}$ \\
\hline
\end{tabular}

\subsection{An Example}

The dataset we use to illustrate our proposal is available in Appendix 1 of Kalbfleisch and Prentice (1980). It is a subset of the data from a randomized trial comparing chemotherapy followed by radiation therapy versus radiation therapy alone in patients with carcinoma of the mouth or throat. The dataset comprises 195 patients and includes follow-up times in days, status at the end of follow-up (dead or censored), and six additional covariates: sex, age at diagnosis, general condition of the patient on a functional rating scale, tumor site, $\mathrm{T}$ stage (size of tumor), and $\mathrm{N}$ stage (extent of nodal metastases). See Zucker (1998) for more details on the dataset and the fitted Cox model stratified by the treatments. For a male subject at age 60.4 (average age across treatment groups) with a tumor site at faucial arch, having Grade category 1, Condition category 2, T staging category 4, and N stage category 0, we plot in Figure 1 the predicted survival difference and the associated simultaneous confidence band on the interval $(81,1100)$. This interval is chosen because there's at least one death in each group beginning from day 81 and the risk set on day 1100 contains around $10 \%$ of subjects in the corresponding group. The critical value $C_{\alpha}$ was estimated to be 8.53 based on 10000 simulations. For this subject, the estimated survival difference is subject to substantial variability as shown by the wide confidence band. The EL confidence band is pretty consistent with the normal confidence band, with only moderate distinction in the early and late ends of the time span. 


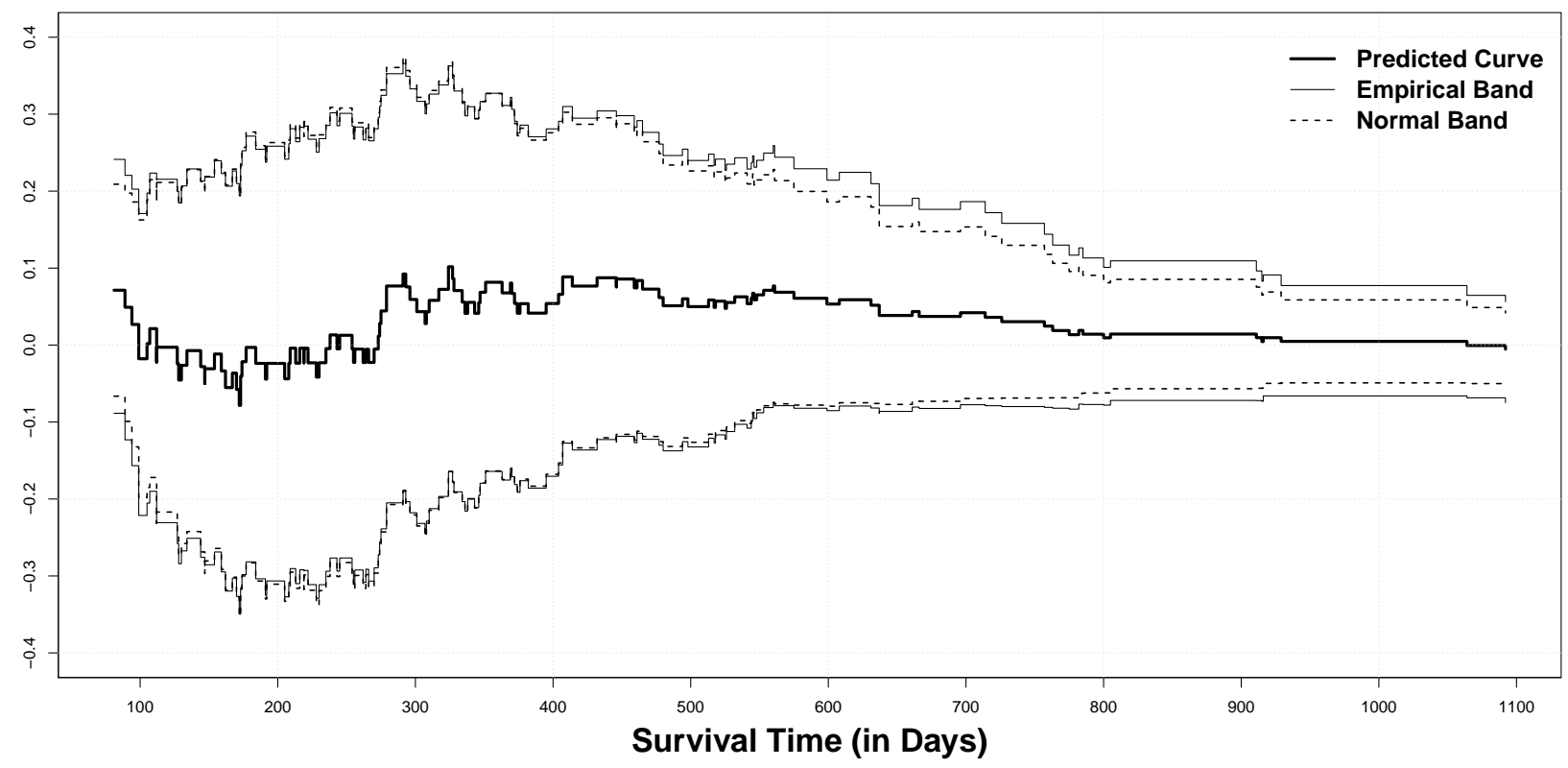

Figure 1: 95\% EL and normal approximation simultaneous confidence bands for the individualized survival difference (Radiation vs. Chemotherapy + Radiation).

\section{Discussion}

The idea in this manuscript is also applicable to other parameters that might be of interest. Particularly, it can be applied to study the difference of two average survival functions defined as $\theta_{0}^{*}(t)=\int_{\boldsymbol{Z}}\left\{S_{1}(t \mid \boldsymbol{Z})-S_{2}(t \mid \boldsymbol{Z})\right\} f(\boldsymbol{Z}) d \boldsymbol{Z}$ (Kim, 2001; Zucker, 1998), where $f(\cdot)$ is the density function of $\boldsymbol{Z}$. This parameter is more relevant if an aggregated difference is desired instead of an individualized one. The example in Kim (2001) shows that this parameter may be comparable with $\theta_{0}\left(\cdot \mid \boldsymbol{Z}_{0}\right)$ in practice, where $\boldsymbol{Z}_{0}$ is the average vector of covariates. For the purpose of EL inference on $\theta_{0}^{*}(t)$, we can fix $\boldsymbol{\beta}$ at $\widehat{\boldsymbol{\beta}}$ and $f(\cdot)$ at its empirical estimator that puts equal weight on each observation, and formulate the following EL ratio at a hypothetical value $\theta^{*}$ and intermediate value $\eta$ :

$$
\Re\left(\theta^{*}, \eta, t\right)=\frac{\sup _{P_{1}, P_{2}}\left\{L\left(P_{1}, P_{2}, \widehat{\boldsymbol{\beta}}\right) \mid P_{1}(t)=-\log \eta, P_{2}(t)=-\log \phi\right\}}{\sup _{\boldsymbol{\beta}, P_{1}, P_{2}} L\left(P_{1}, P_{2}, \boldsymbol{\beta}\right)},
$$

where $\phi=\phi\left(\eta, \theta^{*}\right)$ is given by the equation $n^{-1} \sum_{i=1}^{2} \sum_{j=1}^{n_{i}}\left\{\eta^{\exp \left(\boldsymbol{Z}_{i j}^{\top} \widehat{\boldsymbol{\beta}}\right)}-\phi^{\exp \left(\boldsymbol{Z}_{i j}^{\top} \widehat{\boldsymbol{\beta}}\right)}\right\}=\theta^{*}$. Using techniques similar to those in Section 2, we can analogously derive the asymptotic distribution of this EL ratio and construct the confidence interval and band accordingly.

\section{Acknowledgement}

The authors wish to thank the Associate Editor and a referee for their insightful comments that substantially improved this manuscript. The first author acknowledges support from US NSF grant 1007666. 


\section{Proof}

In what follows, " $O_{p}(\cdot)$ " and " $O(\cdot)$ " are to be understood uniformly in $t \in\left[\tau_{0}, \tau\right]$. Let $\eta_{0}=S_{1}(t)$.

190 Multiplier $\lambda_{i}$ defined in equations (2.5) and (2.6) are of the order $O_{p}\left(n^{-1 / 2}\right)$.

Proof: Without loss of generality, we assume that $T_{i j}$ has been ordered such that $T_{i 1} \leq T_{i 2} \leq \cdots \leq T_{i n_{i}}$. It is then clear that $S_{i 1} \geq S_{i 2} \geq \cdots \geq S_{i n_{i}}$. When $\lambda_{1}>0$, we have

$$
\begin{aligned}
-\log \eta & =\frac{1}{n} \sum_{j=1}^{n_{1}} \frac{\delta_{1 j} g_{1 j}}{S_{1 j}+\lambda_{1}}=\frac{1}{n} \sum_{j=1}^{n_{1}} \frac{\delta_{1 j} g_{1 j}}{S_{1 j}} \frac{S_{1 j}}{S_{1 j}+\left|\lambda_{1}\right|} \\
& \leq \frac{1}{n} \sum_{j=1}^{n_{1}} \frac{\delta_{1 j} g_{1 j}}{S_{1 j}} \frac{S_{11}}{S_{11}+\left|\lambda_{1}\right|}=-\frac{S_{11}}{S_{11}+\left|\lambda_{1}\right|} \log \widehat{S}_{1} .
\end{aligned}
$$

Similarly, when $\lambda_{1}<0$, we have $\log \eta \leq S_{11} /\left(S_{11}-\left|\lambda_{1}\right|\right) \log \widehat{S}_{1}$. Therefore, $\left|\lambda_{1}\right| \leq S_{11}\left|\log \widehat{S}_{1}-\log \eta\right| /|\log \eta|$. The uniform root $n$ consistency of $\widehat{S}_{1}$, uniform boundedness of $S_{11}$ implied by the consistency of $\widehat{\boldsymbol{\beta}}$ and boundedness of $\boldsymbol{Z}_{i j}$, and the assumption that $\left|\eta_{0}-\eta\right| \leq M n^{-1 / 2}$ then imply $\lambda_{1}=O_{p}\left(n^{-1 / 2}\right)$. Moreover, we can rewrite equation (2.5) into

$$
\log \widehat{S}_{1}-\log \eta=-\frac{\lambda_{1}}{n} \sum_{j=1}^{n_{1}} \frac{\delta_{1 j} g_{1 j}}{S_{1 j}^{2}}+\frac{\lambda_{1}^{2}}{n} \sum_{j=1}^{n_{1}} \frac{g_{1 j}}{S_{1 j}^{2}\left(S_{1 j}+\lambda_{1}\right)} .
$$

Note that the first term on the r.h.s. is simply $-\lambda_{1} \widehat{\sigma}_{1}^{2}$. When $g_{1 j}=I\left[T_{1 j} \leq t\right]=1$, we have $T_{1 j} \leq t \leq \tau$, therefore $S_{1 j} \geq S_{i}^{(0)}(\widehat{\boldsymbol{\beta}}, \tau)$ which converges in probability uniformly to $s_{i}^{(0)}\left(\boldsymbol{\beta}_{0}, \tau\right)>0$ by a simple argument using SLLN.

This shows that the second term is of the order $O_{p}\left(n^{-1}\right)$. To conclude,

$$
\log \eta-\log \widehat{S}_{1}=\lambda_{1} \widehat{\sigma}_{1}^{2}+O_{p}\left(n^{-1}\right)
$$

When $\left|\eta-\eta_{0}\right| \leq M n^{-1 / 2}$, there holds $\left|\left(\eta-\theta_{0}\right)-S_{2}\right| \leq M n^{-1 / 2}$, so similarly we have $\lambda_{2}=O_{p}\left(n^{-1 / 2}\right)$ and

$$
\log \left(\eta-\theta_{0}\right)-\log \widehat{S}_{2}=\lambda_{2} \widehat{\sigma}_{2}^{2}+O_{p}\left(n^{-1}\right) .
$$

Lemma 2. With probability approaching one, the likelihood ratio $\Re\left\{\theta_{0}(t), \eta, t\right\}$, as a function of $\eta$, allows a local maximizer $\eta_{n}(t)=\eta_{n}\left\{\theta_{0}(t), t\right\}$ that converges uniformly to $\eta_{0}$.

Proof: For any $\eta$ such that $\left|\eta-\eta_{0}\right| \leq M n^{-1 / 2}$, using Taylor expansion to (2.4) and Lemma 1, we obtain

$$
\begin{aligned}
& -\log \Re\left\{\theta_{0}(t), \eta, t\right\}=\sum_{i=1}^{2} \sum_{j=1}^{n_{i}} \delta_{i j} g_{i j}\left(\frac{\lambda_{i}}{S_{i j}}-\frac{\lambda_{i}^{2}}{2 S_{i j}^{2}}\right)+n \lambda_{1} \log \eta+n \lambda_{2} \log \left(\eta-\theta_{0}\right)+O_{p}\left(n^{-1 / 2}\right) \\
= & n \lambda_{1}\left(\log \eta-\log \widehat{S}_{1}\right)+n \lambda_{2}\left\{\log \left(\eta-\theta_{0}\right)-\log \widehat{S}_{2}\right\}-\frac{n}{2} \sum_{i=1}^{2} \lambda_{i}^{2} \widehat{\sigma}_{i}^{2}+O_{p}\left(n^{-1 / 2}\right) .
\end{aligned}
$$


Due to (A.1), (A.2) and $\log \left(1+M n^{-1 / 2} / S_{1}\right)=O\left(n^{-1 / 2}\right)$, we have

$$
\begin{aligned}
-\log \Re\left(\theta_{0}, \eta_{0}+M n^{-1 / 2}, t\right) & =\sum_{i=1}^{2} \frac{n\left\{\log \left(S_{i}+M n^{-1 / 2}\right)-\log \widehat{S}_{i}\right\}^{2}}{2 \widehat{\sigma}_{i}^{2}}+O_{p}\left(n^{-1 / 2}\right), \\
-\log \Re\left(\theta_{0}, \eta_{0}, t\right) & =\sum_{i=1}^{2} \frac{n\left(\log S_{i}-\log \widehat{S}_{i}\right)^{2}}{2 \widehat{\sigma}_{i}^{2}}+O_{p}\left(n^{-1 / 2}\right) .
\end{aligned}
$$

Note that for $i=1,2$, there holds

$$
\log \left(S_{i}+M n^{-1 / 2}\right)-\log \widehat{S}_{i}=\left(\log S_{i}-\log \widehat{S}_{i}\right)+\log \left(1+M n^{-1 / 2} / S_{i}\right)
$$

The first term on the right hand side is of the order $O_{p}\left(n^{-1 / 2}\right)$ while the second term is of the order $M \cdot O\left(n^{-1 / 2}\right)$, hence by choosing a sufficiently large $M$, the probability that the second term eventually dominates the first term can be made as high as desired. When the second term dominates the first term, we have $\Re\left(\theta_{0}, \eta_{0}+M n^{-1 / 2}, t\right)<$ $\Re\left(\theta_{0}, \eta_{0}, t\right)$. Similar conclusion can be made when we replace $M n^{-1 / 2}$ by $-M n^{-1 / 2}$. This completes the proof.

Proof of the Theorem: Using Taylor expansion to the 1.h.s. of (2.5) and (2.6) at $\left(\eta, \lambda_{1}, \lambda_{2}\right)=\left(\eta_{0}, 0,0\right)$, one can find

$$
\lambda_{i}=\frac{(-1)^{i-1} S_{i}}{S_{1}^{2} \widehat{\sigma}_{1}^{2}+S_{2}^{2} \widehat{\sigma}_{2}^{2}}\left(S_{1} \log \frac{S_{1}}{\widehat{S}_{1}}-S_{2} \log \frac{S_{2}}{\widehat{S}_{2}}\right)+O_{p}\left(n^{-1}\right)
$$

Using (A.1)-(A.3), we obtain

$$
\begin{aligned}
-2 \log \Re\left\{\theta_{0}, \eta_{n}(t), t\right\} & =2 n \lambda_{1}\left\{\lambda_{1} \widehat{\sigma}_{1}^{2}+O_{p}\left(n^{-1}\right)\right\}+2 n \lambda_{2}\left\{\lambda_{1} \widehat{\sigma}_{1}^{2}+O_{p}\left(n^{-1}\right)\right\}-\frac{n}{2} \sum_{i=1}^{2} \lambda_{i}^{2} \widehat{\sigma}_{i}^{2}+O_{p}\left(n^{-1 / 2}\right) \\
& =n \lambda_{1}^{2} \widehat{\sigma}_{1}^{2}+n \lambda_{2}^{2} \widehat{\sigma}_{2}^{2}+O_{p}\left(n^{-1 / 2}\right) .
\end{aligned}
$$

Plugging the expression for $\lambda_{i}$ in (A.4) into the above equality, we have

$$
-2 \log \Re\left\{\theta_{0}, \eta_{n}(t), t\right\}=\frac{n}{S_{1}^{2} \widehat{\sigma}_{1}^{2}+S_{2}^{2} \widehat{\sigma}_{2}^{2}}\left(S_{1} \log \frac{S_{1}}{\widehat{S}_{1}}-S_{2} \log \frac{S_{2}}{\widehat{S}_{2}}\right)^{2}+o_{p}(1) .
$$

It suffices to recall that $\sqrt{n}\left(S_{1} \log S_{1} / \widehat{S}_{1}-S_{2} \log S_{2} / \widehat{S}_{2}\right) \rightarrow U(t)$ in distribution (Zhang and Klein, 2001).

\section{References}

Bie O, Borgan $\varnothing$, Liestøl K. Confidence intervals and confidence bands for the cumulative hazard rate function and their small sample properties. Scandinavian Journal of Statistics 1987;14(3):221-33.

Dong B, Matthews DE. Empirical likelihood for cumulative hazard ratio estimation with covariate adjustment. Biometrics 2012;68(2):408-18.

Einmahl JH, McKeague IW. Confidence tubes for multiple quantile plots via empirical likelihood. The Annals of Statistics 1999;27(4):1348-67. 
Hjort NL, McKeague IW, Van Keilegom I. Extending the scope of empirical likelihood. The Annals of Statistics 2009;37(3):1079-111.

Kalbfleisch JD, Prentice RL. The statistical analysis of failure time data. New York: John Wiley \& Sons, 1980.

Kim J. Confidence intervals for the difference of median survival times using the stratified cox proportional hazards model. Biometrical Journal 2001;43(6):781-90.

Lin D, Fleming T, Wei L. Confidence bands for survival curves under the proportional hazards model. Biometrika 1994;81(1):73-81.

McKeague IW, Zhao Y. Simultaneous confidence bands for ratios of survival functions via empirical likelihood. Statistics \& Probability Letters 2002;60(4):405-15.

Naik-Nimbalkar U, Rajarshi M. Empirical likelihood ratio test for equality of k medians in censored data. Statistics \& Probability Letters 1997;34(3):267-73.

Parzen M, Wei L, Ying Z. Simultaneous confidence intervals for the difference of two survival functions. Scandinavian Journal of Statistics 1997;24(3):309-14.

Shen J, He S. Empirical likelihood for the difference of two survival functions under right censorship. Statistics \& Probability Letters 2006;76(2):169-81.

Wang Q, Wang JL. Inference for the mean difference in the two-sample random censorship model. Journal of Multivariate Analysis 2001;79(2):295-315.

Zhang MJ, Klein JP. Confidence bands for the difference of two survival curves under proportional hazards model. Lifetime Data Analysis 2001;7(3):243-54.

Zhao Y, Zhao M. Empirical likelihood for the contrast of two hazard functions with right censoring. Statistics \& Probability Letters 2011;81(3):392-401.

Zucker DM. Restricted mean life with covariates: modification and extension of a useful survival analysis method. Journal of the American Statistical Association 1998;93(442):702-9. 